W stużbie tradycji i odnowy liturgicznej. 50 lat Instytutu Liturgicznego w Krakowie (1968-2018), red. P. Nowakowski, J. Mieczkowski, Kraków 2019, s. 171-192.

ISBN 978-83-7438-849-8 (wersja drukowana), ISBN 978-83-7438-850-4 (wersja online) DOI:http://dx.doi.org/10.15633/9788374388504.15

János Nyirán Instytut Teologiczny Świętego Atanazego w Nyíregyháza, Węgry

\title{
Da una prassi sbagliata fino al rinnovamento del servizio diaconale nella tradizione della Chiesa Greco-Cattolica Ungherese
}

Nella storia della Chiesa Greco-cattolica Ungherese c'è stato un abuso reiterato per decenni che consisteva nell'assistenza liturgica da parte dei seminaristi, i quali svolgevano il ruolo e i compiti del diacono e il suddiacono senza aver ricevuto la chirotonia o la chirotesia ${ }^{1}$. Questo vuol dire che i seminaristi svolgevano a turno il servizio diaconale, o servivano come suddiaconi, anche se in verità quest'ultimi assumevano in questo modo le funzioni del secondo diacono. Risulta che quest'usanza era diffusa anche nelle Diocesi Greco-cattoliche limitrofe, ed era già presente ai tempi dell'erezione dell'Eparchia di Hajdúdorog (1912).

Lo scopo della mia presentazione è far conoscere i dettagli di questa prassi liturgica illegittima dimostrando e valutando oggettivamente, per

1 Come si distingue oggi generalmente l'imposizione delle mani per l'ordine sacro (chirotonia) e per gli ordini minori (chirotesia). 
quanto sia possibile, i suoi vantaggi e svantaggi, anche perché fino ad oggi vi sono ancora alcuni sacerdoti e ricercatori che ritengono che quest'usanza sia una pratica molto utile, anzi necessaria, per il nostro tempo.

Il divieto di tale pratica abusiva avvenne solo nel 1993, quando il Vescovo Szilárd Keresztes la proibì con un decreto episcopale ${ }^{2}$.

D'altro canto, invece, voglio presentare, come una posizione totalmente contrastante, il rinnovamento del servizio diaconale che è incominciata nella Chiesa Greco-cattolica Ungherese quasi alla fine del primo millennio.

\section{Status questionis}

Anche se prima tanti sacerdoti erano convinti dell'utilità e della necessità della menzionata pratica liturgica, finora solo un sacerdote studioso si è prodigato a raccogliere i dati storici sulla questione in oggetto. Si tratta del Rev. István Ivancsó, docente presso 1'Istituto Teologico Greco-cattolico Sant' Atanasio; egli è un liturgista che studia soprattutto le fonti liturgiche ungheresi. I suoi studi sulla tematica comunque sono a favore del ripristino della pratica dell'assistenza liturgica dei seminaristi, perciò gli studi finora fatti devono essere riveduti ${ }^{3}$.

2 Cf. Archivio Eparchiale Greco-cattolico, Prot. N. 267/1993 del 18 febbraio 1993, e Prot. N. 784/1993 del 13 maggio 1993.

3 Cf. I. Ivancsó, Asszisztencia [Assistenza], in: "Egy szájjal és egy szívvel". A Szent Atanáz Görög Katolikus Hittudományi Főiskola Jubileumi Emlékkönyve (1950-200o) [Con una bocca e con un cuore - Albo Giubilare dell'Istituto Teologico Superiore Greco-cattolico di Sant'Atanasio (1950-2000)], a cura di I. Ivancsó, Nyíregyháza 2000, p. 157-164 (in seguito: I. Ivancsó, Assistenza); I. Ivancsó, Egy régi dokumentum az asszisztálásról [Un documento antico sull'assistenza liturgica], in: "Athanasiana" 12 (2001), p. 155-160 (in seguito: I. Ivancsó, Un documento antico); I. Ivancsó, A kispapok asszisztálása a magyar görög katolikus egyházban [L'assistenza dei chierici nella Chiesa greco-cattolica ungherese], in: Ecclesiam aedificans. A 70 éves Keresztes Szilárd püspök köszöntése [Ecclesiam aedificans. Saluto del Vescovo Szilárd Keresztes di 70 anni], a cura di I. Ivancsó, Nyíregyháza 2002, p. 155-181 (in seguito: I. Ivancsó, L'assistenza dei chierici); I. Ivancsó, A magyar görög katolikus kispapok oltárszolgálata [The Seminarians of the Hungarian Byzantine Catholic Church as Acolytes and their Service], in: "Posztbizánci Közlemények" V (2002), p. 66-85, 153-154 (I. Ivancsó, The Seminarians); I. Ivancsó, L'assistenza dei chierici nella 
Negli articoli di P. Ivancsó troviamo una buona presentazione dello status questionis, di cui citiamo solo i risultati più importanti.

\title{
1.1. Una lettera circolare del Vescovo János Pásztélyi Kovács
}

\author{
Prima di tutto P. Ivancsó cita una lettera circolare di S.E. Mons. János \\ Pásztélyi Kovács, Vescovo dell’Eparchia di Mukacevo dal 1874 fino al 1891, \\ il cui contenuto si cita di seguito:
}

Miei figli diletti in Cristo,

Reverendissimi Sotto-arciprete e Sacerdoti,

Sperimentando con dolore dalla parte del governo della diocesi che alcuni sacerdoti trascurano l'ufficio dei Vespri e dei Mattutini domenicali e festivi e i piccoli funerali che sono senza pagamento, ed affidano il compimento di tale ufficiature al cantore o ai seminaristi che sono da loro. Ed i chierici hanno il coraggio di vestirsi con il Felonio e l'Epitrachelion durante queste occasioni. Volendo per il futuro abolire tale prassi, si prescrive quanto segue:

I. Ogni sacerdote deve celebrare i Mattutini e i Vespri (utrenya - vecsernye) domenicali e festivi ed ogni funerale senza ritardo e con grande cura.

II. Per i seminaristi è proibito severamente indossare i paramenti di Felonio-epitrachelion e celebrare i sacri uffici dei Mattutini, Vespri e funerali. Chi fa il contrario e chi glieli permette, sarà punito con una pena adeguata ${ }^{4}$.

III. Durante i sacri uffici possono assistere solo i seminaristi tonsurati.

IV. Gli arcipreti e sotto-arcipreti sono obbligati a vigilare sull'osservanza precisa della presente prescrizione e coloro che ricoprono uffici di responsabilità devono denunciare coloro che non osservano tale prescrizione.

Con la mia benedizione episcopale, dato in Uzhgorod, il 28 giugno 1875.

Chiesa greco-cattolica ungherese, "Folia Athanasiana" 5 (2003), p. 19-41 (in seguito: I. Ivancsó, L'assistenza dei chierici nella Chiesa greco-cattolica ungherese); I. Ivancsó, Kispapi asszisztálás a magyar görög katolikus egyházban - forrásbemutatás I-II [L'assistenza dei seminaristi nella Chiesa greco-cattolica ungherese - ricerca delle fonti I-II], “Athanasiana” 30 (2009), p. 21-57; 31 (2010), p. 9-36 (in seguito: I. Ivancsó, L'assistenza dei seminaristi I-II).

4 Qua I. Ivancsó fa una traduzione più letterale: "Chi fa il contrario e chi glieli permette, attribuisce al suo coraggio le conseguenze più gravi del procedimento realizzabile più serio". Si veda: I. Ivancsó, L'assistenza dei chierici nella Chiesa greco-cattolica ungherese, p. 25. 


\section{Ai miei diletti}

il vostro Padre in Cristo:

Vescovo János 5 .

Questa lettera circolare è molto severa, perché rivela che alla fine del XIX secolo vi erano gravissimi abusi nel servizio liturgico. In questo periodo i seminaristi espletavano anche servizi propri dell'ordine sacerdotale, che tale prassi costituiva certamente una gravissima anomalia, che fin dall'inizio dell'episcopato del Vescovo János Pásztélyi Kovács viene rimproverata e proibita.

Tuttavia, dalla lettera risulta evidente che ai seminaristi tonsurati viene permesso di compiere il servizio diaconale senza riserva, anche se nel documento non si descrivono i dettagli dell'assistenza.

\subsection{Il documento dello svolgimento dell'assistenza nel 1932/1933}

La prima documentazione con una descrizione precisa risale al 1932, quando i seminaristi greco-cattolici del Seminario Centrale di Budapest chiesero con una lettera al Vescovo István Miklósy di approvare la loro regola circa l'assistenza liturgica durante la Divina Liturgia ${ }^{6}$. In questo periodo la maggior parte dei seminaristi greco-cattolici studiava a Budapest, dove avevano la possibilità di frequentare la parrocchia greco-cattolica e celebrare la Divina Liturgia quasi come diaconi. Perciò era necessario avere una descrizione precisa sia dei testi, sia dei movimenti liturgici riguardanti il servizio dei seminaristi praticato in chiesa. Effettivamente questa è la prima compilazione in lingua ungherese che fissa il servizio già in uso. Il permesso dalla parte del Vescovo Miklósy arriva con alcune correzioni nel 19337. Purtroppo ne è rimasto sono un esemplare frammentario che è molto usato ${ }^{8}$.

5 A Hajdúdorogi Külhelynökség Levéltára [Archivio del Vicariato Foraneo di Hajdúdorog], Prot. N. 212/1875 del 28 giugno 1875.

6 Cf. Archivio Eparchiale Greco-cattolico, Prot. N. 2495/1932 del 17 novembre 1932.

7 Cf. Archivio Eparchiale Greco-cattolico, Prot. N. 2216/1933 del 24 ottobre 1933.

8 Cf. I. Ivancsó, Un documento antico 155-16o; I. Ivancsó, The Seminarians 66-85, p. 153-154. 


\subsection{Il documento dello svolgimento dell'assistenza nel 1955}

La seguente testimonianza è una compilazione fatta dal seminarista György Virányi nel 1955, quando frequentava il quinto anno dell'Istituto Teologico, diventato sacerdote successivamente (e ormai in pensione). Quest'opera non ha un permesso scritto dal Vescovo, ma è stata conservata in buono stato, perciò la possiamo esaminare nella sua integrità?.

Proprio per questa ragione ho scelto questo testo per fare un paragone tra il servizio diaconale e quello che allora poteva fare un seminarista. In una tabella vengono elencati i movimenti dei "seminaristi-diaconi" descritti nel documento sopracitato del 1955 facendo un confronto con l'uso attuale.

\subsubsection{Vespri}

\begin{tabular}{|c|c|c|}
\hline Vespri & Nel 1955 & Nel 2018 \\
\hline \multirow[t]{2}{*}{ Paramenti liturgici } & $\begin{array}{l}\text { Vespri e Mattutini: } \\
\text { dalmatica }^{10} \text {, orarion }\end{array}$ & \multirow[t]{2}{*}{$\begin{array}{l}\text { sticharion, orarion, } \\
\text { epimanichia (polsini) }\end{array}$} \\
\hline & $\begin{array}{l}\text { Divina Liturgia: amitto, } \\
\text { sticharion, cintura, epima- } \\
\text { nichia, dalmatica, orarion }\end{array}$ & \\
\hline Grande litania & diacono & $1^{\circ}$ diacono \\
\hline Benedizioni dell'incenso & Diacono: Benedici, signore... & $\begin{array}{l}1^{\circ} \text { diacono: Benedici, } \\
\text { signore... }\end{array}$ \\
\hline Ingresso con l'incensiere & diacono & $1^{\circ}$ diacono \\
\hline Sapienza! In piedi! & diacono & $1^{\circ}$ diacono \\
\hline Incensazione & diacono & $1^{\circ}$ diacono \\
\hline $\begin{array}{l}\text { Intonazione prima del } \\
\text { prokimenon }\end{array}$ & $\begin{array}{l}\text { Diacono: Sapienza! } \\
\text { Sacerdote: Pace a tutti! Sud- } \\
\text { diacono: Stiamo attenti! }\end{array}$ & $\begin{array}{l}1^{\circ} \text { diacono: Sapienza! } \\
\text { Sacerdote: Pace a tutti! } \\
2^{\circ} \text { Diacono: Stiamo attenti! }\end{array}$ \\
\hline Prima delle letture & $\begin{array}{l}\text { Diacono: Sapienza! } \\
\text { Suddiacono: Stiamo attenti! }\end{array}$ & $\begin{array}{l}1^{\circ} \text { diacono: Sapienza! } \\
2^{\circ} \text { diacono: Stiamo attenti! }\end{array}$ \\
\hline
\end{tabular}

9 La trascrizione (in lingua ungherese) si veda: I. Ivancsó, L'assistenza dei seminaristi I, p. 21-57; II, p. 9-36.

10 Lo sticharion del diacono che è decorato secondo il colore liturgico prescritto. Risulta di particolare interesse che generalmente si usa la forma: il vestito del diacono che usa durante la liturgia festiva. 


\begin{tabular}{|l|l|l|}
\hline Ectenie: Diciamo tutti... & Diacono & $1^{\circ}$ diacono \\
\hline Ectenie: Compiamo... & Suddiacono & $2^{\circ}$ diacono \\
\hline Inchinate il vostro capo... & Suddiacono & $1^{\circ}$ diacono \\
\hline Sapienza! & Diacono & $1^{\circ}$ diacono \\
\hline
\end{tabular}

\subsubsection{I mattutini}

\begin{tabular}{|c|c|c|}
\hline Mattutini & Nel 1955 & Nel 2018 \\
\hline \multirow[t]{2}{*}{ Paramenti liturgici } & $\begin{array}{l}\text { Vespri e Mattutini: } \\
\text { dalmatica, orarion }\end{array}$ & \multirow[t]{2}{*}{$\begin{array}{l}\text { sticharion, orarion, } \\
\text { epimanichia (polsini) }\end{array}$} \\
\hline & $\begin{array}{l}\text { Divina Liturgia: amitto, } \\
\text { sticharion, cintura, epima- } \\
\text { nichia, dalmatica, orarion }\end{array}$ & \\
\hline Grande litania & Diacono & $1^{\circ}$ diacono \\
\hline Il Signore è Dio... & - & - \\
\hline 3 piccole litanie & - & diaconi a turno \\
\hline $\begin{array}{l}\text { Intonazione prima del } \\
\text { prokimenon }\end{array}$ & $\begin{array}{l}\text { Diacono: Sapienza! } \\
\text { Sacerdote: Pace a tutti! } \\
\text { Suddiacono: Stiamo attenti! }\end{array}$ & $\begin{array}{l}1^{\circ} \text { diacono: Sapienza! } \\
\text { Sacerdote: Pace a tutti! } \\
2^{\circ} \text { Diacono: Stiamo attenti! }\end{array}$ \\
\hline $\begin{array}{l}\text { Incensazione con le ectenie } \\
\text { prima del Vangelo }\end{array}$ & Diacono & $1^{\circ}$ diacono \\
\hline Sapienza! In piedi! & Diacono & $1^{\circ}$ diacono \\
\hline Stiamo attenti! & Suddiacono & $2^{\circ}$ Diacono \\
\hline Salva, o Dio, il tuo popolo... & Diacono & $1^{\circ}$ Diacono \\
\hline $\begin{array}{l}\text { Durante il canone } \\
\text { piccole litanie }\end{array}$ & - & diaconi a turni \\
\hline $\begin{array}{l}\text { Incensazione e Magnifichia- } \\
\text { mo la Madre-di-Dio... }\end{array}$ & Diacono & $\begin{array}{l}2 \text { diaconi } \\
2^{\circ} \text { diacono }\end{array}$ \\
\hline Santo è il Signore & Suddiacono & $1^{\circ}$ diacono \\
\hline $\begin{array}{l}\text { Gloria a te che ci hai mostrato } \\
\text { la luce }\end{array}$ & Diacono & Sacerdote \\
\hline Ectenie: Diciamo tutti... & Diacono & $1^{\circ}$ diacono \\
\hline Ectenie: Compiamo... & Suddiacono & $2^{\circ}$ diacono \\
\hline Inchinate il vostro capo... & Suddiacono & $1^{\circ}$ diacono \\
\hline Sapienza! & Diacono & $1^{\circ}$ diacono \\
\hline
\end{tabular}




\subsubsection{La Divina Liturgia}

\begin{tabular}{|c|c|c|}
\hline Divina Liturgia & Nel 1955 & Nel 2018 \\
\hline \multirow[t]{2}{*}{ Paramenti liturgici } & $\begin{array}{l}\text { Vespri e Mattutini: } \\
\text { dalmatica, orarion }\end{array}$ & \multirow[t]{2}{*}{$\begin{array}{l}\text { sticharion, orarion, } \\
\text { epimanichia (polsini) }\end{array}$} \\
\hline & $\begin{array}{l}\text { Divina Liturgia: amitto, } \\
\text { sticharion, cintura, epima- } \\
\text { nichia, dalmatica, orarion }\end{array}$ & \\
\hline Incensazione & Diacono & Diaconi \\
\hline Benedici, signore & Diacono & $1^{\circ}$ Diacono \\
\hline Grande litania & Diacono & $1^{\circ}$ Diacono \\
\hline Piccole litanie & - & diaconi a turno \\
\hline Ingresso con l'Evangeliario & Diacono & $1^{\circ}$ Diacono \\
\hline $\begin{array}{l}\text { Benedici, signore, il santo } \\
\text { Ingresso! }\end{array}$ & Diacono & $1^{\circ}$ Diacono \\
\hline Sapienza! In piedi! & Diacono & $1^{\circ}$ Diacono \\
\hline $\begin{array}{l}\text { E nei secoli dei secoli-prima } \\
\text { del Trisaghion }\end{array}$ & Diacono & $1^{\circ}$ Diacono \\
\hline $\begin{array}{l}\text { Intonazione prima del } \\
\text { prokimenon }\end{array}$ & $\begin{array}{l}\text { Diacono: Sapienza! } \\
\text { Sacerdote: Pace a tutti! Sud- } \\
\text { diacono: Stiamo attenti! }\end{array}$ & $\begin{array}{l}1^{\circ} \text { Diacono: Sapienza! } \\
\text { Sacerdote: Pace a tutti! } \\
2^{\circ} \text { Diacono: Stiamo attenti! }\end{array}$ \\
\hline Incensazione & Diacono & Diaconi \\
\hline Benedici, Signore... & Diacono & $1^{\circ}$ Diacono \\
\hline $\begin{array}{l}\text { Sapienza! In piedi! Ascoltiamo } \\
\text { il santo Vangelo. }\end{array}$ & Suddiacono & $1^{\circ}$ Diacono \\
\hline $\begin{array}{l}\text { Lettura del Santo Vangelo } \\
\text { secondo N. }\end{array}$ & Diacono & $1^{\circ}$ Diacono \\
\hline Stiamo attenti! & Suddiacono & $2^{\circ}$ Diacono \\
\hline Ectenie: Diciamo tutti... & Diacono & $1^{\circ}$ Diacono \\
\hline Incensazione & Diacono & Diaconi \\
\hline Grande Ingresso & $\begin{array}{l}\text { Diacono con l'incensiere, } \\
\text { Suddiacono con l'aerion }\end{array}$ & $\begin{array}{l}1^{\circ} \text { Diacono con il diskos } \\
\text { e con l'aerion sulla spalla, } \\
2^{\circ} \text { Diacono con l'incensiere }\end{array}$ \\
\hline Il Signore Dio si ricordi... & Diacono & $1^{\circ}$ Diacono \\
\hline $\begin{array}{l}\text { Dialogo tra il sacerdote } \\
\text { e il diacono }\end{array}$ & - & + \\
\hline
\end{tabular}




\begin{tabular}{|l|l|l|}
\hline $\begin{array}{l}\text { Amiamoci gli uni gli altri... } \\
\text { Le porte! Le porte!... Stiamo } \\
\text { con devozione... }\end{array}$ & Diacono & $1^{\circ}$ Diacono \\
\hline Asteriscos & Non si parla & $1^{\circ}$ Diacono lo toglie \\
\hline $\begin{array}{l}\text { Elevazione del disco } \\
\text { e calice }\end{array}$ & Non si parla & $1^{\circ}$ Diacono \\
\hline Incensazione & Diacono & Diaconi \\
\hline $\begin{array}{l}\text { Ectenie: Ricordando tutti } \\
\text { i santi... }\end{array}$ & Suddiacono & $\begin{array}{l}\text { Comincia il } 1^{\circ} \text { Diacono, } \\
\text { e la conclude il } 2^{\circ} \text { Diacono }\end{array}$ \\
\hline Inchinate il vostro capo... & Suddiacono & $1^{\circ}$ Diacono \\
\hline Stiamo attenti! & Suddiacono & $1^{\circ}$ Diacono \\
\hline Con timore di Dio & Diacono & $1^{\circ}$ Diacono \\
\hline $\begin{array}{l}\text { In piedi! Dopo aver parteci- } \\
\text { pato... }\end{array}$ & Diacono & $1^{\circ}$ Diacono \\
\hline Procediamo in pace! & Diacono & Sacerdote \\
\hline Preghiamo il Signore! & Diacono & $2^{\circ}$ Diacono \\
\hline Sapienza! & Diacono & $1^{\circ}$ Diacono \\
\hline
\end{tabular}

\subsubsection{Alcune osservazioni}

Esaminando attentamente le tabelle possiamo costatare che nella descrizione dei Vespri e dei Mattutini, vi sono piccolissime differenze tra il servizio del seminarista assistente e quello del diacono. Nella presentazione della Divina Liturgia vi sono invece alcune differenze, tra cui quelle più rilevanti riguardano il servizio legato ai sacri vasi liturgici. I seminaristi secondo le descrizioni del loro sevizio non toccano i sacri vasi, mentre i diaconi sono obbligati a ciò. Un'altra dissomiglianza nel documento del 1955 è l'omissione dei dialoghi tra il sacerdote e il diacono, che nell'attuale prassi liturgica vengono regolarmente recitati. Gli altri esempi non risultano avere una grande importanza degna di menzione.

Riassumendo tutto ciò possiamo concludere che il servizio dell'assistenza fatto dai seminaristi negli anni '50, e si suppone anche negli anni successivi, è quasi conforme a quello diaconale eccetto per le azioni che implicavano di prendere in mano (toccare) i vasi sacri. 


\section{Valutazione della pratica dell'assistenza}

Dopo quest'affermazione dobbiamo valutare se questo servizio dei seminaristi fosse veramente così utile e talmente necessario da essere usato per lungo tempo.

\subsection{Argomenti in favore della pratica dell'assistenza}

Nei diversi articoli P. Ivancsó ha raccolto tanti argomenti a favore dell'assistenza ed alcuni contro di essa, ma come ho già notato prima questi argomenti devono essere riveduti a causa della loro unilateralità ${ }^{11}$.

2.1.1. Conoscenza più sicura delle ufficiature

L'autore fa una distinzione tra l'insegnamento teoretico e quello pratico, sottolineando l'importanza di quel pratico, ed afferma che con questa pratica liturgica in un certo senso cresce anche la responsabilità del seminarista, che impara bene lo svolgimento delle ufficiature, e nello stesso tempo se ne affeziona facilmente ${ }^{12}$.

Commento: Il seminarista deve imparare tantissime cose durante gli anni della sua formazione, anche quelle pratiche riguardanti il servizio liturgico. Deve imparare a fare il cantore - per diventare lettore - a fare il chierichetto - per diventare suddiacono - durante le Divine Liturgie, i Vespri, i Mattutini, alla presenza di un sacerdote, o di molti sacerdoti concelebranti, oppure sotto la presidenza di un Vescovo. D'altra parte, l'insegnamento della Teologia non comincia con esegesi biblica, oppure con teologia dogmatica, perché queste materie richiedono altre materie fondamentali, infatti, prima, si deve imparare la base su cui si può costruire. $\mathrm{Ci}$ vuole una progressione continua anche nel servizio liturgico. Quando qualcuno sta per avvicinarsi al diaconato è sufficiente imparare i movimenti propri del diacono durante la Liturgia. Per di più attualmente l'esercizio del diaconato non dura più solamente $2-4$ giorni come nel passato (quando

\footnotetext{
11 Perciò prima presento riassumendo gli argomenti di P. Ivancsó, e poi subito faccio il mio commento.

12 Cf. I. Ivancsó, Assistenza, p. 161; I. Ivancsó, L'assistenza dei chierici, p. 176; I. Ivancsó, The Seminarians, p. 79; I. Ivancsó, L'assistenza dei chierici nella Chiesa greco-cattolica ungherese, p. 36-37.
} 
generalmente il 2 agosto aveva luogo l'ordinazione diaconale e il 6 agosto successivo quella sacerdotale). Oggi il servizio diaconale in Ungheria dura da 6 a 12 mesi, e questo è un periodo del tutto sufficiente per imparare bene le funzioni e i movimenti propri del diacono o del sacerdote.

\subsubsection{Apprendimento dei movimenti liturgici}

Padre Ivancsó afferma che i diaconi e i sacerdoti ordinati senza la pratica dell'assistenza commettono molto errori nello svolgimento delle ufficiature. L'autore fa menzione anche del passato quando il Vescovo Imre Timkó voleva edificare una cappella per i seminaristi, che durante il regime comunista era un'idea irreale, perciò aveva chiesto l'autorizzazione delle autorità civili per costruire "un campo liturgico di addestramento". In questo modo riuscì ad ottenere il permesso statale. Secondo P. István proprio questa funzione della cappella ha perso il suo significato o scopo originale ${ }^{13}$.

Commento: Prima di tutto dobbiamo costatare che dal nome inventato ingegnosamente dal Vescovo Timkó non dobbiamo fare grandi conclusioni, perché era semplicemente una mistificazione/evasione del regime anticlericale. E comunque una cappella o chiesa non può essere mai un campo di addestramento a causa delle sacre ufficiature che ivi vengono celebrate. D'altra parte i seminaristi devono imparare i movimenti liturgici, però non durante i sacri uffici, ma in tempo opportuno, come avevamo già notato sopra, e nei nostri giorni (grazie ai tre nuovi vescovi) è divenuto impensabile ricevere l'ordinazione presbiterale senza aver prima esercitato il servizio diaconale per un certo periodo.

L'apprendimento e la comprensione della Divina Liturgia e delle ufficiature sacre da parte del chierico (diaconi, sacerdoti o vescovi) non si esaurisce in un periodo di tempo. Dobbiamo studiarle, capirle e meditarle sempre più profondamente. In questo senso non si può dire che il seminarista ha imparato la liturgia tre-quattro anni. E se riconosciamo che dobbiamo studiare continuamente la celebrazione delle ufficiature o almeno durante i nostri 20, 50 o 70 anni del sacerdozio, allora possiamo affermare tranquillamente che la pratica dell'assistenza appresa durante gli anni trascorsi in seminario non costituisce un vantaggio realistico.

13 Cf. I. Ivancsó, Assistenza, p. 161; I. Ivancsó, L'assistenza dei chierici, p. 176; I. Ivancsó, The Seminarians, p. 79; I. Ivancsó, L'assistenza dei chierici nella Chiesa greco-cattolica ungherese, p. 37. 
Ci si domanda se un seminarista può servire come diacono, allora il sacerdote perché non può celebrare come un vescovo? Entrambi i casi sono inammissibili, e tutto ciò è assolutamente proibito dalle norme della Chiesa.

2.1.3. Crescita della cultura musicale

Coloro che esercitavano la pratica dell'assistenza (nel 2000 - quando è stato scritto l'articolo in questione - erano i seminaristi di nazionalità ungherese dall'estero, soprattutto quelli dell'Eparchia di Mukacevo, che studiavano ancora a Nyíregyháza) avevano una cultura musicale molto più elevata rispetto ai seminaristi dell’Eparchia di Hajdúdorog o dell'Esarcato Apostolico di Miskolc, scrive P. István nel suo articolo ${ }^{14}$.

Commento: Quest'affermazione è assolutamente soggettiva, perché da un lato la cultura musicale dipende dalla persona stessa, e se durante i sei anni del Seminario uno può imparare melodie liturgiche difficili, come p. es. i canoni o gli stichirá idiomela, allora sarà capace di istruirsi molto facilmente con il canto delle litanie o ektenie, poiché la melodia di quest'ultime sono molto facili. Se invece qualcuno non sa cantare, con la semplice pratica può raggiungere un livello accettabile.

2.1.4. Educazione al maggior zelo

Un seminarista se serve all'altare e fa la pratica dell'assistenza diventa più disciplinato, poiché la vicinanza dell'altare educa l'inserviente ${ }^{15}$.

Commento: $\mathrm{Si}$, è vero, è un bell'assioma dell'autore. Ma se il seminarista fa il servizio di chierichetto, allora serve vicino all'altare, e nel senso dell'assioma delineato può diventare ugualmente disciplinato. D'altra parte, come abbiamo già notato sopra, per l'apprendimento della Divina Liturgia ci vuole sempre un progresso graduale.

14 Cf. I. Ivancsó, Assistenza, p. 161; I. Ivancsó, L'assistenza dei chierici, p. 176; I. Ivancsó, The Seminarians, p. 79-80; I. Ivancsó, L'assistenza dei chierici nella Chiesa greco-cattolica ungherese, p. 37 .

15 Cf. I. Ivancsó, Assistenza, p. 161; I. Ivancsó, L'assistenza dei chierici, p. 176; I. Ivancsó, The Seminarians, p. 80; I. Ivancsó, L'assistenza dei chierici nella Chiesa greco-cattolica ungherese, p. 37. 


\subsubsection{Animazione vocazionale}

I giovani ammiravano i seminaristi che facevano la pratica dell'assistenza, e di conseguenza secondo Padre Ivancsó questa pratica poteva suscitare nuove vocazioni ${ }^{16}$.

Commento: Grazie a Dio vi sono ancora tra i giovani coloro che ammirano i seminaristi, e possono partecipare liberamente anche oggi - anzi nel nostro tempo non vi sono impedimenti come durante il tempo del comunismo - a incontri di ogni genere, dove grazie alla presenza dei seminaristi possono essere attratti dalla vita sacerdotale. I seminaristi veramente girano tanto, organizzano tanti programmi facendo tante conoscenze ed amicizie soprattutto con i giovani. Inoltre, i tre Vescovi della Chiesa Metropolitana sui iuris d'Ungheria nelle loro visite pastorali alle parrocchie sovente sono accompagnati dai seminaristi della propria Eparchia per fare il servizio come suddiaconi e lettori durante la Liturgia Pontificale. Quindi, anche in questo modo possono essere un buon esempio per i giovani.

\subsubsection{Aiuto ai sacerdoti}

È noto che il servizio sacerdotale è abbastanza sovraccarico, perciò i seminaristi possono aiutare tanto con la loro pratica dell'assistenza, soprattutto ai sacerdoti più anziani - scrive così P. Ivancsó ${ }^{17}$.

Commento: Sì e no. Sì, perché i seminaristi possono aiutare in tantissimi luoghi e in svariate situazioni: ospedali, ricoveri per anziani, scuole, con i disabili, i bambini ciechi, ed altro. In realtà, il corso di formazione prevede un anno di esperienza sociale al termine del secondo anno di filosofia. D’altra parte, vi sono i diaconi, i quali possono collaborare con i sacerdoti e con i Vescovi sia per il servizio liturgico, che nei diversi ambiti socio-caritativi e pastorali.

\subsubsection{Incremento della solennità}

Padre Ivancsó afferma che è stato diminuito grandemente la solennità delle Liturgie pontificali con il divieto della pratica dell'assistenza dei se-

16 Cf. I. Ivancsó, Assistenza, p. 161-162; I. Ivancsó, L'assistenza dei chierici, p. 177; I. Ivancsó, The Seminarians, p. 80; I. Ivancsó, L'assistenza dei chierici nella Chiesa greco-cattolica ungherese, p. 37.

17 Cf. I. Ivancsó, Assistenza, p. 162; I. Ivancsó, L'assistenza dei chierici, p. 177; I. Ivancsó, The Seminarians, p. 80; I. Ivancsó, L'assistenza dei chierici nella Chiesa greco-cattolica ungherese, p. 37. 
minaristi avvenuto nel 1993, anzi in occasione delle feste patronali anche i fedeli risentono della mancanza dei seminaristi assistenti ${ }^{18}$.

Commento: Forse veramente vi era un periodo quando le Liturgie Pontificali avevano un carattere più solenne, ma anche in quel tempo era necessario il servizio di almeno sei seminaristi ( 2 per trikirion e dikirion, 2 per la mitra e il pastorale, e due per l'incenso). Se vi era ancora un diacono, allora il numero degli inservienti aumentava. Dal 2008 invece questo problema non si è più avuto perché la Liturgia Pontificale è diventata molto complessa e solenne, tantoché alcuni sacerdoti ritengono che sia un'esagerazione.

\subsubsection{Aumento della devozione dei fedeli}

Scrivendo sullo zelo dei fedeli P. Ivancsó affermando che nelle parrocchie in cui ha lavorato, quando abitava anche un seminarista o vi faceva assistenza liturgica i parrocchiani erano molto più entusiasti e frequentavano più volentieri la chiesa perché potevano ammirare che un loro parente svolgeva il servizio liturgico ${ }^{19}$.

Commento: Quanto sopra affermato lascia intendere che alcuni fedeli si recavano in chiesa solo per ammirare il modo di celebrare del loro parente seminarista. Ma certamente questo motivo non sembra essere un detonante per accrescere la religiosità o la devozione, quanto piuttosto il numero della loro presenza.

2.1.9. Più grandi collette in chiesa

È un fatto che là dove esisteva l'assistenza, i fedeli hanno sempre portato un'offerta più grande - scrive P. Ivancsó, ed aggiunge ancora nel punto 10.

2.1.10. Raccolte più abbondanti per il Seminario

Quando esisteva l'assistenza, i fedeli erano più generosi anche nelle raccolte per il Seminario ${ }^{20}$.

18 Cf. I. Ivancsó, Assistenza, p. 162; I. Ivancsó, L'assistenza dei chierici, p. 177; I. Ivancsó, The Seminarians, p. 80; I. Ivancsó, L'assistenza dei chierici nella Chiesa greco-cattolica ungherese, p. 37. 19 Cf. I. Ivancsó, Assistenza, p. 162; I. Ivancsó, L'assistenza dei chierici, p. 177; I. Ivancsó, The Seminarians, p. 80; I. Ivancsó, L'assistenza dei chierici nella Chiesa greco-cattolica ungherese, p. 37. 20 Cf. I. Ivancsó, Assistenza, p. 162; I. Ivancsó, L'assistenza dei chierici, p. 177; I. Ivancsó, The Seminarians, p. 80; I. Ivancsó, L'assistenza dei chierici nella Chiesa greco-cattolica ungherese, p. 38. 
Commento: Gli ultimi due argomenti trattano dell'aspetto economico, che sono molto importanti per la gestione del Seminario, ma se la somma delle elemosine è diminuita non è del tutto sicuro che la causa sia stata l'abolizione della pratica dell'assistenza dei seminaristi. Le raccolte di beni materiali sicuramente sono diventate più limitate sia perché meno persone si dedicano all'agricoltura, sia per la dilagante scristianizzazione che inizia a colpire anche la nostra realtà.

\subsection{Argomenti contro la pratica dell'assistenza}

Dopo ciò P. Ivancsó raccoglie alcuni argomenti contrari alla pratica dell'assistenza da parte dei seminaristi, ma precisa che essi sono di gran lunga minori rispetto alle ragioni che supportano la pratica abusiva in oggetto ${ }^{21}$.

2.2.1. Chi non è stato ordinato diacono non può esercitare le funzioni diaconali

Riconoscendo il fatto indubitabile - come scrive l'Autore stesso: - ossia "i servizi sono legati al sacro ordine" oppure: "Le persone elette e provate sono state ordinate al servizio adeguato". Dopo queste affermazioni però l'Autore cerca altre anomalie per offuscare il vero significato giuridico dell'Ordine sacro, e pone una domanda - secondo le sue parole - "grave" dicendo:

i nostri cantori senza l'ordine minore di lettore leggono quotidianamente la prima lettura dell'Apostolo Paolo, questo non è da considerarsi un abuso? Questo è adatto alla tradizione cosiddetta orientale?22

Commento: Dobbiamo rispondere subito che le due questioni non sono allo stesso livello. Se un seminarista fa il servizio diaconale commette un grave abuso contro gli Ordini sacri, ma nell'altro caso invece (se un cantore svolge il suo servizio senza vere ricevuto l'ordine minore del lettorato)

21 Cf. I. Ivancsó, Assistenza, p. 162; I. Ivancsó, L'assistenza dei chierici, p. 178; I. Ivancsó, The Seminarians, p. 81; I. Ivancsó, L'assistenza dei chierici nella Chiesa greco-cattolica ungherese, p. 38. 22 I. Ivancsó, Assistenza, p. 162-163; I. Ivancsó, L'assistenza dei chierici, p. 178; I. Ivancsó, The Seminarians, p. 81; I. Ivancsó, L'assistenza dei chierici nella Chiesa greco-cattolica ungherese, p. 38-39. 
non possiamo affermare una cosa simile. Il secondo paragrafo del canone 408 del Codex canonum Ecclesiarum Orientalium [CCEO] dice al riguardo:

§ 2. (cf 228 § 2) Oltre agli incarichi ecclesiastici, ai quali i laici sono ammessi dal diritto comune, essi possono essere assunti dall'autorità competente anche ad altri incarichi, eccettuati quelli che richiedono l'ordine sacro o che sono espressamente vietati ai laici dal diritto particolare della propria Chiesa sui iuris.

Nello stesso tempo bisogna notare che i Vescovi attuali cercano di recuperare anche la dignità degli ordini minori, e ormai vi sono dei cantori i quali hanno già ricevuto l'ordine minore del lettorato.

Come se fosse una circostanza attenuante l'Autore continua che negli ultimi tempi

Prima della proibizione dell'assistenza, per lungo era in uso la consuetudine che il chierico assistente non leggeva il vangelo, né indossava l'orarion (il segno dell'ordine proprio diaconale), né tantomeno poteva esercitare altre funzioni diaconali (neanche prima), perché non gli era permesso toccare i vasi sacri ${ }^{23}$.

Tuttavia, nel documento analizzato nei paragrafi precedenti risulta il contrario di quanto si afferma qui sopra.

\subsubsection{Esercizi dei riti interi}

I seminaristi potevano celebrare i Vespri e i Mattutini interamente, omettendo ovviamente le benedizioni sacerdotali. Tale pratica "veramente non si adatta alla tradizione della Chiesa bizantina, ma è stata di grande aiuto per i sacerdoti che avevano dei chierici nelle loro parrocchie" - scrive P. Ivancsó ${ }^{24}$.

Commento: nel corso dei secoli anche il rito bizantino ha conosciuto degli sviluppi che hanno portato alla forma attuale. Ad esempio nel primo millennio anche gli Horologhia sono stati compilati senza prevedere necessariamente la presenza di un sacerdote per la celebrazione delle ufficiature

23 I. Ivancsó, Assistenza, p. 163; I. Ivancsó, L'assistenza dei chierici, p. 178; I. Ivancsó, The Seminarians, p. 81; I. Ivancsó, L'assistenza dei chierici nella Chiesa greco-cattolica ungherese, p. 38-39.

24 Cf. I. Ivancsó, Assistenza, p. 163; I. Ivancsó, L'assistenza dei chierici, p. 178-179; I. Ivancsó, The Seminarians, p. 81; I. Ivancsó, L'assistenza dei chierici nella Chiesa greco-cattolica ungherese, p. 39. 
(difatti, non sempre nei monasteri c'erano ieromonaci), tuttavia in qualche modo potevano celebrare ${ }^{25}$. È molto evidente che i seminaristi nel nostro tempo non possono fare ugualmente - come rafforza anche il nostro Auto$\mathrm{re}^{26}$, ma risulta molto strano che neanche i diaconi non possono celebrare alcuna ufficiatura da soli, eccetto le piccole ore. I nostri Vescovi sono molto coerenti su questa questione ${ }^{27}$.

2.2.3. La richiesta della dispensa dalle irregolarità

"Le persone, che volevano ricevere l'ordinazione, dovevano richiedere la dispensa dalle irregolarità per l'esercizio non autorizzato dei sacri servi$\mathrm{zi}^{\prime \prime}$ - scrive molto brevemente questa volta l'Autore.

Commento: Qua non vi è nessun'appello da parte dell'Autore, anche perché tutto ciò era evidente dalla consuetudine della Chiesa greco-cattolica Ungherese $^{28}$, anche se il CCEO riguardo agli impedimenti di tale caso afferma solamente questo:

È impedito dal ricevere gli ordini sacri $[\ldots]$ chi ha posto un atto di ordine riservato a chi è costituito nell'ordine dell'episcopato o del presbiterato 29 .

\subsubsection{La pratica della Chiesa ortodossa}

P. Ivancsó a questo punto fa una certa critica contro la Chiesa ortodossa ed anche contro quei sacerdoti greco-cattolici ai quali piacciono le usanze ortodosse. Da cui consegue l'antipatia dell'autore contro gli ortodossi, ed infatti, afferma che i greco-cattolici devono isolarsi dalle pratiche ortodosse.

25 Cf. E. Velkovska, Libri liturgici bizantini, in: Scientia liturgica, Manuale di liturgia I, direzione di J. A. Chupungco, Casale Monferrato 1998, p. 243-258.

26 Cf. I. Ivancsó, Assistenza, p. 163; I. Ivancsó, L'assistenza dei chierici, p. 179; I. Ivancsó, The Seminarians, p. 81; I. Ivancsó, L'assistenza dei chierici nella Chiesa greco-cattolica ungherese, p. 39.

27 P. es. i Vescovi non lasciano celebrare i Vespri e i Mattutini nemmeno al diacono nostro che vive a Sopron, in ambiente latino, anche se il suo parroco vive a Győr (circa 90 chilometri di distanza).

28 Non ancora diritto particolare, poiché quello si sta preparando nei nostri giorni per la Chiesa Metropolitana sui iuris d'Ungheria.

29 CCEO, can. $762 \S 1$. 
E poi arriva a dire se questa pratica dell'assistenza da parte dei seminaristi non è conosciuta per diverse ragioni nella Chiesa ortodossa, allora i greco-cattolici la possono esercitare, perché non devono seguire categoricamente gli ortodossi ${ }^{30}$.

Commento: In questo luogo volevo menzionare tutta la polemica di P. Ivancsó contro gli ortodossi, con i quali noi in Ungheria non abbiamo mai avuto grandi problemi, discussioni o litigi, mantenendo in genere buoni rapporti con loro. Questa situazione favorevole è probabilmente dovuta alla loro scarsità numerica ed anche al fatto che i loro fedeli appartengono a diverse nazionalità. Per quanto sopra non sembra opportuno entrare in dispute particolari.

Dall'altra parte dobbiamo sottolineare che nell' Istruzione per l'applicazione delle prescrizioni liturgiche del Codice dei Canoni delle Chiese Orientali vi sono diversi riferimenti ai fratelli ortodossi. In particolare, nel capitolo 21, che porta il titolo: Valore ecumenico del patrimonio liturgico comune ${ }^{31}$, si afferma che:

In ogni sforzo di rinnovamento liturgico si dovrà pertanto tenere conto della prassi dei fratelli Ortodossi, conoscendola, stimandola ed allontanandosene il meno possibile per non accrescere le separazioni esistenti, ma anzi intensificando gli sforzi in vista di eventuali adattamenti, da maturare ed operare congiuntamente. Si manifesterà così l'unità che già sussiste nel ricevere quotidianamente la stessa linfa spirituale proveniente dall'esercizio del comune patrimonio ${ }^{2}$.

\subsection{Valutazione}

P. István Ivancsó nei suoi articoli riguardanti l'assistenza dei seminaristi, richiama sempre l'attenzione al fatto che tale pratica "risale a tempo imme-

30 Cf. I. Ivancsó, Assistenza, p. 163-164; I. Ivancsó, L'assistenza dei chierici, p. 179; I. Ivancsó, The Seminarians, p. 81-82; I. Ivancsó, L'assistenza dei chierici nella Chiesa greco-cattolica ungherese, p. 39.

31 Istruzione per l'applicazione delle prescrizioni liturgiche del Codice dei Canoni delle Chiese Orientali, Città del Vaticano 1996, p. 21.

32 Giovanni Paolo II, Discorso ai partecipanti alla riunione sui problemi pastorali della Chiesa cattolica di rito bizantino in Romania (22 gennaio 1994), "L'Osservatore Romano", 22 gennaio 1994, p. 5; vedi anche in "Servizio Informazioni per le Chiese Orientali" 49 (1994), p. 2. 
morabile e pertanto può essere considerata una consuetudine, che però nel 1993 è stata proibita da un decreto episcopale"33. Questa dichiarazione non è da trascurare poiché il Canone 6 del CCEO dice:

Con l'entrata in vigore del Codice:

$1^{\circ}$ sono abrogate tutte le leggi di diritto comune o di diritto particolare che sono contrarie ai canoni del Codice, oppure che riguardano una materia che è stata integralmente ordinata nel Codice;

$2^{\circ}$ sono revocate tutte le consuetudini che sono riprovate dai canoni del Codice, oppure quelle che sono contrarie a essi, ma non le centenarie o immemorabili.

Tenendo conto del menzionato N. 2 del Can. 6, e se si considera che l'assistenza dei chierici nella liturgia fosse una consuetudine centenaria o immemorabile, allora essa non poteva essere revocata.

Tuttavia, bisogna anche tenere presente che prima dell'entrata in vigore del Codice, vi erano state alcune proibizioni da parte della Santa Sede circa la pratica in questione, come scrive pure P. Ivancsó 34 :

Nel 1953 la Congregazione per le Chiese Orientali in un'istruzione liturgica ha affermato: "Nefas est clerico qui Ordinem diaconatus non acceperint munere diaconali fungi" 35 . Tale divieto viene reiterato anche nel paragrafo in cui si tratta dell'uso dei paramenti liturgici: "Nemini licet induere vestem sacram quae propria est Ordini superiori eo quem habet" ${ }^{36}$.

33 I. Ivancsó, Assistenza, p. 159; I. Ivancsó, L'assistenza dei chierici, p. 155; I. Ivancsó, Un documento antico, p. 155-156; I. Ivancsó, The Seminarians, p. 71; I. Ivancsó, L'assistenza dei chierici nella Chiesa greco-cattolica ungherese, p. 19.

34 Cf. I. Ivancsó, L'assistenza dei chierici, p. 174-175; I. Ivancsó, L'assistenza dei chierici nella Chiesa greco-cattolica ungherese, p. 35-36.

35 Sacra Congreatione pro Ecclesia Orientali, Ordo celebrationis vesperarum, matutini ed Divinae Litugiae iuxta recensionem Ruthenorum, Romae 1953, 11, 26: "I chierici che non hanno preso il diaconato non possono servire nella carica diaconale".

36 Sacra Congreatione pro Ecclesia Orientali, Ordo celebrationis vesperarum..., 10, 24: “Non è lecito a nessuno indossare la veste sacra propria dell'ordine maggiore". Benché non sia strettamente conforme al nostro tema è degno di osservare anche un altro punto di vista. Era una pratica nella Chiesa greco-cattolica ungherese che nell'interesse dell'aumento della solennità della Santa Liturgia (oppure nelle Liturgie pontificali) un sacerdote 
Tali prescrizioni non sono state ben accolte in Ungheria, tant'è che la prassi "antica" dell'assistenza è continuata ad essere in uso anche dopo l'entrata in vigore del Codex Canonum Ecclesiarium Orientalium nel 1991, ritenendo la prassi come una consuetudine immemorabile (ex can. 6, n. 2). Ad ogni modo, S. E. Mons. Szilárd Keresztes, nel 1993, chiese in merito un parere alla Congregazione per le Chiese Orientali ${ }^{37}$, il cui responso definiva la pratica in questione come una consuetudine contraria al Diritto e quindi da vietare. Il decreto di proibizione è stato promulgato successivamente anche a seguito di un fatto di cui il Vescovo venne a conoscenza, ossia le esequie di un seminarista celebrate dai suoi compagni di corso.

Circa la tematica in questione si ritiene opportuno menzionare che il Tit. 75 dell'Istruzione per l'applicazione delle prescrizioni liturgiche del Codice dei Canoni delle Chiese Orientali, pubblicato dalla Congregazione per le Chiese Orientali, esorta a "favorire un reale e coerente esercizio degli Ordini".

Gli Ordini minori e il diaconato non sono una pura formalità in vista dell'ordinazione presbiterale. Essi abilitano a un preciso servizio nella Chiesa, e come tali vanno effettivamente esercitati, in forma definitiva per coloro che non intendono accedere al presbiterato, in forma sufficientemente ampia per quanti saranno ordinati presbiteri. Ciò vale in modo particolare per il diaconato. In questo senso non si tema di conferire gli Ordini minori ed anche il diaconato a quanti, di buoni costumi, debitamente preparati e adatti al compito che assumono, si dichiarano disponibili al servizio della Chiesa, anche se dovessero continuare a vivere in famiglia e ad esercitare il proprio mestiere. In questo modo si otterranno anche i ministri necessari ad un decoroso svolgimento della liturgia, evitando la prassi, mutuata anch'essa dalla Chiesa latina ed ora in essa non più in uso, di far esercitare a ministri di rango superiore la funzione liturgica che sarebbe riservata a quelli di rango inferiore (il caso più frequente è quello di presbiteri che fungono da

esercitava la funzione diaconale. Sembra che questo - come l'assistenza dei chierici - non fosse solo la caratteristica della nostra Chiesa. Prof. Daniele Gelsi specialista della Liturgia menzionava tra gli abusi liturgici citava anche quello dei sacerdoti che in interesse dell'aumento della solennità esterna fanno servizio diaconale nella Liturgia. Cf. D. Gelsi, Orientali, Liturgie, in: Nuovo dizionario di liturgia, a cura di D. Sartore, a. m. Triacca, Roma² 1984, p. 1004 .

37 Cf. Archivio Eparchiale Greco-cattolico, Prot. N. 179/1993 del 21 gennaio 1993. 
diaconi), o di affidare stabilmente a laici compiti liturgici che spettano a un ministro: prassi, queste, da eliminare ${ }^{8}$.

Per quanto sopra va evitato di

1. far esercitare a ministri di rango superiore la funzione liturgica che sarebbe riservata a quelli di rango inferiore (il caso più frequente è quello di presbiteri che fungono da diaconi),

2. o di affidare stabilmente a laici compiti liturgici che spettano a un ministro.

Questo passo è valido non solo agli accoliti o ai laici latini, i quali distribuiscono la comunione, ma anche ai seminaristi greco-cattolici che praticano il servizio diaconale. In pari tempo, il menzionato documento suggerisce il recupero del servizio diaconale.

\section{Rinnovamento del servizio diaconale}

Il punto 76 dell'Istruzione parla del diaconato nei termini seguenti:

Il diaconato è stato istituito non per il sacerdozio ma per il servizio dei Vescovi e dei presbiteri. I diaconi erano infatti, un tempo considerati come la mano o l'occhio di questi; oppure, secondo la formula di Ignazio di Antiochia, in armonia con essi manifestano al popolo fedele "il comandamento del Signore" 39 .

Simile prospettiva, conservata nelle Chiese ortodosse e in via di recupero nelle comunità latine, va rimessa in piena luce anche nelle Chiese orientali cattoliche. Il ripristino della sua missione liturgica ed extraliturgica appare infatti, di grande utilità ${ }^{40}$.

38 Istruzione per l'applicazione delle prescrizioni liturgiche del Codice dei Canoni delle Chiese Orientali, p. 61.

39 Ignazio di Antiochia, Lettera agli Smirnesi VIII, 1: P. Th. Camelot, Ignace d'Antioche, Polycarpe de Smyrne. Lettres. Martyre de Polycarpe, in Sources Chrétiennes 1o bis, Paris² 1951, p. $162-163$.

40 Cf. Istruzione per l'applicazione delle prescrizioni liturgiche del Codice dei Canoni delle Chiese Orientali, p. 62. 
S. E. Mons. Szilárd Keresztes non ha fatto tradurre il testo dell'Istruzione, ma lo conosceva molto bene, e teneva in gran conto l'importanza e l'utilità del servizio diaconale, perciò nel 1999 ha chiesto a due seminaristi ritornati da Roma di fare un servizio diaconale per 1 anno (è da notare che Padre Ivancsó proprio in questo tempo cominciava la ricerca sull'assistenza liturgica dei seminaristi). Successivamente, nel 2001, il Presule ha ordinato un altro seminarista che ha svolto il servizio diaconale per più di due anni. Dopo una piccola pausa, nel 2005, lo scrivente ha ricevuto l'ordine sacro del diaconato, che continua ad esercitare fino ad oggi. Nell'ultimo decennio il numero dei diaconi nella Chiesa greco-cattolica ungherese è aumentato, e ad oggi se ne contano ben venti.

\begin{tabular}{|l|l|l|l|}
\hline 2018 & $\begin{array}{l}\text { Arcieparchia } \\
\text { di Hajdúdorog }\end{array}$ & Eparchia di Miskolc & $\begin{array}{l}\text { Eparchia } \\
\text { di Nyíregyháza }\end{array}$ \\
\hline Diaconi permanenti & 6 & 2 & 1 \\
\hline $\begin{array}{l}\text { Diaconi che si } \\
\text { preparano al sacer- } \\
\text { dozio }\end{array}$ & 6 & 0 & 5 \\
\hline Somma & 12 & 2 & 6 \\
\hline Somma totale & $\mathbf{2 0}$ & & \\
\hline
\end{tabular}

Si può pertanto affermare che nell'attualità il servizio diaconale è stato ripristinato è non si presenta alcuna necessità di rispristinare una pratica abusiva come quella dell'assistenza liturgica dei seminaristi. D'altra parte sarebbe impensabile riproporre una simile prassi, attesa la sua illegittimità suffragata dallo stesso CCEO e dai Documenti ufficiali della Sede Apostolica, nonché dalle istruzioni in merito date dalla congregazione per le Chiese Orientali. 


\section{Streszczenie}

\section{Od błędnej praktyki do odnowy postugi diakonatu w tradycji Kościoła greckokatolickiego na Wegrzech}

W historii Kościoła greckokatolickiego na Węgrzech istniał dziwny rodzaj asysty liturgicznej ze strony kleryków. (W tym miejscu musimy odnotować, że zwyczaj ten był rozpowszechniony w sąsiadujących diecezjach greckokatolickich).

Pierwsze dokumenty sięgają 1932 roku, kiedy to klerycy greckokatoliccy z Centralnego Seminarium w Budapeszcie poprosili w liście do biskupa Istvána Miklósyego o przyjęcie ich reguły dotyczącej asysty liturgicznej podczas świętej liturgii. W tym okresie większość kleryków greckokatolickich studiowała w Budapeszcie, gdzie mieli oni możliwość uczęszczania do kościoła greckokatolickiego i sprawowania świętej liturgii prawie tak samo jak diakoni. Tak więc istniała konieczność posiadania opisu zarówno tekstów, jak i czynności liturgicznych dotyczących służby kleryków praktykowanej w kościele.

Celem mojego referatu jest zapoznanie ze szczegółami tej praktyki liturgicznej oraz ukazanie i ocenienie, na ile to możliwe w sposób obiektywny, jej zalet i wad, również dlatego, że do dziś są jeszcze kapłani i naukowcy, którzy uważają ten zwyczaj za bardzo przydatną praktykę, wręcz konieczną w naszych czasach, a taka praktyka była już obecna przy ustanawianiu eparchii Hajdúdorogu (1912).

Ponadto chcę przedstawić kwestię odnowy służby diakonalnej, która rozpoczęła się w Kościele greckokatolickim na Węgrzech pod koniec pierwszego tysiąclecia. 